\title{
Neue Technologien im öffentlichen Sektor: Bürgerinnen und Bürger haben nur geringe Erwartungen.
}

\author{
Caroline Fischer, Jurgen Willems, Joachim Van den Bergh
}

\section{Preprint-Version}

\section{Publiziert in:}

Fischer, Caroline; Willems, Jurgen; Van den Bergh, Joachim (2021): Was erwarten Bürger:innen von der Nutzung neuer Technologien im öffentlichen Sektor? Nicht viel. In: Verwaltung und Management 27 (2), S. 59 - 67, DOI: https://doi.org/10.5771/0947-9856-2021-2-59.

\section{Abbildung frei verfügbar und wiederverwendbar:}

Willems, Jurgen; Fischer, Caroline; Van den Bergh, Joachim (2021a): Citizens' Expected Impact of New Technologies in Public and For-profit Services. Auswertungen und Abbildungen. Online verfügbar unter https://doi.org/10.6084/m9.figshare.13693693.

\section{Daten frei verfügbar und wiederverwendbar:}

Willems, Jurgen; Fischer, Caroline; Van den Bergh, Joachim (2021b): Citizens' Expected Impact of New Technologies in Public and For-profit Services. Datensatz. Online verfügbar unter https://osf.io/vqtgc.

\begin{abstract}
Aktuelle technologische Entwicklungen (z.B. künstliche Intelligenz) führen immer wieder zu Debatten, wie diese sinnvoll für die öffentliche Verwaltung genutzt werden können. Die Verwaltung selbst und entsprechende Expertinnen und Experten diskutieren mögliche positive Wirkungen auch in Bezug auf Werte, wie Effizienz, Transparenz oder Fairness. Diese Studie untersucht, ob Bürgerinnen und Bürger von der Anwendung dieser Technologien in der öffentlichen Verwaltung ebensolche Wirkungen ebenso erwarten. Dabei wird auch analysiert, ob eine andere Erwartungshaltung als bei gewinnorientierten Unternehmen besteht. Die Befragungsergebnisse ( $\mathrm{N}=1.577)$ zeigen, dass weder negative noch positive Erwartungen in Bezug auf Wirkungen dieser Technologien bestehen, das gilt für öffentliche wie für die privatwirtschaftliche Leistungserbringung. Es wird diskutiert, ob die Ergebnisse in der bisher mangelnden Anwendung der untersuchten Technologien begründet liegen oder aus einer unzureichenden Kommunikation mit und Einbindung von Bürgerinnen und Bürgern in entsprechende Planungs- und Implementationsprozesse resultieren.
\end{abstract}




\section{Hintergrund und Fragestellung}

Forschung und Beratung im Bereich E-Government und Digitalisierung der Verwaltung haben immer wieder auf die Potenziale dieser Veränderungen hingewiesen. Digitalisierung wird mit einer höheren Effizienz, einer besseren Leistungserbringung, größerer Transparenz, mehr Partizipations- und Kollaborationsmöglichkeiten für Bürgerinnen und Bürger in Verbindung gebracht (Heuermann et al. 2018; Lindgren et al. 2019). Dennoch können Studien diese Erwartungen bisher nicht empirisch aufzeigen und es bleibt oft bei der bloßen Formulierung von Zielen.

Forschung zu Digitalisierung stellt stattdessen häufig den Status Quo einzelner Reformen oder Veränderungsprozesse dar (Boudreau und Bernier 2017; Cavalheiro und Joia 2016; Mergel 2019), bestimmt Erfolgskriterien (Andersen et al. 2011; Große Starmann und Wiedemann 2017; Schmid 2019) oder untersucht die Akzeptanz der beteiligten Stakeholder (Alruwaie et al. 2020; Dekker et al. 2020; Wagner 2018). Das scheint vor allem daran zu liegen, dass Technologie sich kontinuierlich weiterentwickelt und zu neuen Digitalisierungsprojekten, Prozessüberarbeitungen oder neuen digitalen Leistungen führt. Endergebnisse, im Sinne von Wirkungen, hingegen sind schwer zu bestimmen.

Aktuelle technologische Entwicklungen im Kontext künstlicher Intelligenz, dem Internet der Dinge, der Nutzung von Robotern oder der Blockchain-Technologie führen immer wieder zu Debatten, wie diese sinnvoll für die öffentliche Verwaltung genutzt werden können, und zwar über den Selbstzweck der technologischen Innovation hinaus ${ }^{1}$. Die Verwaltung und entsprechende Expertinnen und Experten erhoffen sich hier positive Wirkungen in Bezug auf die Planung und das Monitoring von Verwaltungsprozessen, Entlastung der Mitarbeitenden, insbesondere in Entscheidungsprozessen, und eine verbesserte Interaktion mit Bürgerinnen und Bürgern (Engelmann und Puntschuh 2020; Willems et al. 2017).

Beispiele für die Anwendung solcher Technologien sind im deutschen Verwaltungskontext oft noch nicht verbreitet, existieren aber zumindest in Pilotprojekten. Beispielsweise wurde die BlockchainTechnologie (eine dezentrale Datenbankstruktur, in der das Teilnehmernetzwerk Daten verwaltet), in einem Projekt des Bundesamtes für Migration und Flüchtlingen (BAMF) verwendet, um sensible personenbezogene Daten von Asylsuchenden behördenübergreifend auszutauschen (Guggenmos et al. 2019). Algorithmen werden häufig als Assistenzsysteme für eine Entscheidungsfindung genutzt, beispielsweise im sogenannten predictive policing. Ein konkretes Beispiel ist das Assistenzsystem AMAS des österreichischen Arbeitsmarktservice, das aufgrund persönlicher Merkmale einer arbeitssuchenden Person analysiert, wie wahrscheinlich diese Person auf dem Arbeitsmarkt vermittelbar ist. Auf Basis der so errechneten Vermittlungswahrscheinlichkeit werden den Arbeitssuchenden bestimmte Qualifikationsmaßnahmen angeboten (Puntschuh und Fetic 2020, S. 12). Ein Beispiel für lernende Systeme (künstliche Intelligenz) stellt der Chatbot Bobbi der Berliner Verwaltung dar. Dieser

\footnotetext{
${ }^{1}$ Siehe zum Beispiel: Die Zeit: Mit künstlicher Intelligenz zum Kita-Platz https://www.zeit.de/2021/06/kuenstliche-intelligenz-kita-anwendung-regulierung-gesellschaft-technologie
} 
virtuelle Assistent liefert Bürgerinnen und Bürgern in einem Live-Chat Informationen zu Verwaltungsleistungen. Aus bereits erfolgten Gesprächen lernt der Chatbot dabei für zukünftige Nutzeranfragen und die Passfähigkeit der vorgeschlagenen Antworten (Puntschuh und Fetic 2020, S. 14). Die Stadt Wien bietet einen solchen Chatbot sogar als App an, die auch Wege beschreiben und Fahrplanauskünfte geben kann (Wienbot). ${ }^{2}$

Der Grad der tatsächlichen Verwendung dieser Technologien, die über die bloße Digitalisierung von Akten oder die digitale Abwicklung von Prozessen hinaus gehen, unterscheidet sich stark zwischen einzelnen Staaten und sogar innerhalb dieser. Während Staaten wie Estland (Anthes 2015) oder Taiwan (Linders et al. 2018) auf einer relativ weit vorangeschrittenen Stufe der Digitalisierung agieren (sog. maturity level, Coursey \& Norris, 2008), sind andere immer noch dabei von Papierakten auf elektronische Alternativen umzustellen oder schaffen gerade das Fax als Kommunikationskanal ab. Von diesem Stand dürfte auch stark abhängen, welche (mittel- oder langfristigen) Wirkungen von Digitalisierung überhaupt schon zu beobachten sind.

Abgesehen von der Anwendung der genannten Technologien und deren Wirkung auf die unmittelbare Leistungserbringung der Verwaltung muss auch immer wieder hinterfragt werden, welche Wirkung zum Guten oder Schlechten - diese technologischen Weiterentwicklungen in Bezug auf grundlegende gesellschaftliche Werte (sog. public values) entfalten (Jørgensen und Bozeman 2007; Twizeyimana und Andersson 2019). Hierbei geht es um Werte wie erschwingliche öffentliche Dienstleistungen, zeitnahe Leistungserbringung, Schutz von Daten von Bürgerinnen und Bürgern, Transparenz von Staat und Verwaltung sowie die ethisch verantwortungsvolle und faire Behandlung von Bürgerinnen und Bürgern. Diese Werte stehen zum Teil auch konträr zueinander und können nicht immer gleichzeitig erfüllt werden (mit einem Fokus auf Transparenz: Fischer und Kraus 2020; Vanderelst und Willems 2020).

Der oben beschriebene Chatbot kann zum Beispiel durch eine einfachere Verfügbarkeit mehrerer Sprachen erreichen, was in der persönlichen Beratung oft nicht möglich ist: die Zugänglichkeit von Verwaltungsleistungen für Nicht-Muttersprachlerinnen und -Muttersprachler verbessern. Die oben angesprochene Nutzung von Blockchains durch das BAMF lässt die Bearbeitung von Asylanträgen zeitund kostensparender werden und ist auch für die Antragstellerinnen und Anstragsteller transparenter (Schulze et al. 2019). Der von der österreichischen Arbeitsvermittlung genutzte Algorithmus hingegen wurde medial als intransparent und diskriminierend diskutiert und zeitweilig von der zuständigen Datenschutzbehörde gestoppt (Puntschuh und Fetic 2020). Hier standen sich (von außen wahrgenommene) Transparenz und Qualität der Leistungserbringung sowie Nachteile für Einzelne und gesamtgesellschaftliche Vorteile jeweils diametral gegenüber (Fanta 2020; z.B. Köver 2019, 2020).

Offen ist dennoch, wie Bürgerinnen und Bürger im Unterschied zu Expertinnen und Experten die Wirkungen dieser Technologien wahrnehmen. Diese Erwartungshaltung der Bürgerinnen und Bürger

\footnotetext{
${ }^{2}$ Bobbi: https://service.berlin.de/chatbot/chatbot-bobbi-606279.php; Wienbot: https://www.wien.gv.at/bot.
} 
ist aber zentral: Denn wenn Steuergelder in technologischen Fortschritt investiert werden, muss den Steuerzahlerinnen und Steuerzahlern auch vermittelt werden, wofür diese gut sind. Die Frage ist also, ob Bürgerinnen und Bürger den Aufwand, der aktuell in technologische Entwicklungen gesteckt wird, eigentlich wertschätzen. Um diesen Wandel nachhaltig fortzusetzen, sollte auf eine breite Unterstützung durch Bürgerinnen und Bürger gesetzt werden, der die Veränderungen legitimiert und weitere Investitionen rechtfertigt. Abgesehen davon muss gerade technologischer Wandel auf Nutzerorientierung setzen, um in eine qualitativ hochwertige Leistungserbringung zu münden (Róiste 2013). Die Erwartungshaltung von Nutzerinnen und Nutzern technologiebasierter Leistungen sollte daher ergründet und mit den intendierten Wirkungen von Veränderungen verglichen werden (Scupola und Zanfei 2016).

Die vorliegende Studie will daher zunächst klären, welche Wirkungen Bürgerinnen und Bürger von neuen Technologien in Bezug auf gesellschaftliche Werte erwarten.

Darüber hinaus sind public values nicht nur für die Leistungserbringung der öffentlichen Verwaltung, sondern auch für die Serviceleistungen privatwirtschaftlicher Unternehmen von Relevanz. Auch hier erwarten Nutzerinnen und Nutzer Datenschutz, faire Behandlung oder verantwortungsvolle Ressourcenbeschaffung (Franke et al. 2013; Kennedy et al. 2017; Woldaregay et al. 2020), z.T. schreiben dies entsprechende gesetzliche Regelungen sogar vor (z.B. Datenschutzgrundverordnung DSGVO). Aufgrund der vollkommen anderen Natur und Zielstellung öffentlicher Dienstleistungen die Bereitstellung basaler öffentlicher Güter - dürfte sich die Erwartungshaltung der Bürgerinnen und Bürger an diese im Vergleich zu Dienstleistungen und Produkten privater Unternehmen aber unterscheiden. Werte wie Gleichbehandlung, Transparenz oder Partizipation sollten von öffentlichen Organisationen in einem höheren Maße erwartet werden (Behnke 2005).

Die vorliegende Studie untersucht daher ergänzend die Frage, inwiefern sich die Erwartungshaltung der Bürgerinnen und Bürger bzw. Kundinnen und Kunden in Bezug auf die Wirkungen neuer Technologien unterscheidet, je nachdem ob diese Technologien im öffentlichen oder privatwirtschaftlichen Kontext verwendet werden.

\section{Methode und Datenbasis}

Im November 2020 wurde eine Online-Befragung unter deutschen Bürgerinnen und Bürgern durchgeführt (N=1.577). Dabei wurde der Panel-Anbieter Qualtrics genutzt. Die Stichprobe der Befragten ist repräsentativ für die deutsche Bevölkerung in Bezug auf die Altersverteilung und das Geschlecht sowie repräsentativ über alle Bundesländer verteilt. Tabelle 1 zeigt eine genauere Verteilung demografischer Merkmale innerhalb der Stichprobe. Die Bürgerinnen und Bürger wurden gefragt, ob sie davon ausgehen, dass aktuelle neue technologische Entwicklungen (künstliche Intelligenz, Internet der Dinge, Maschinenlernen, Robotisierung und Blockchain) einen negativen oder positiven Einfluss 
auf zehn verschiedene Aspekte öffentlicher und privatwirtschaftlicher Leistungserbringung haben und wie stark sie diesen einschätzen (Effizienz, Erschwinglichkeit von Leistungen, Qualität von Dienstleistungen, faire Behandlung, Sicherheit, allgemeine Zufriedenheit mit Leistung, Transparenz, Datenschutz, ethisch verantwortungsvolle Behandlung, allgemeines Wohlbefinden). Diese zehn Aspekte spiegeln zentrale Werte, im Sinne von public values, wider.

Tabelle 1: Charakteristika der Stichprobe, $\mathrm{N}=1.577$

\begin{tabular}{llll} 
& & N & Proz. Anteil \\
\hline \multirow{3}{*}{ Alter in Jahren } & Unter 30 & 281 & $17.82 \%$ \\
& $30-45$ & 381 & $24.16 \%$ \\
& $45-60$ & 417 & $26.44 \%$ \\
& Über 60 & 498 & $31.58 \%$ \\
\hline \multirow{3}{*}{ Geschlecht } & weiblich & 811 & $51.43 \%$ \\
& männlich & 766 & $48.57 \%$ \\
\hline \multirow{3}{*}{ Beschäftigungsstatus } & Student:in & 106 & $6.89 \%$ \\
& Angestellte:r & 719 & $46.75 \%$ \\
& Rentnëndige:r & 99 & $6.44 \%$ \\
& arbeitslos & 447 & $29.06 \%$ \\
& privatwirtschaftlich & 441 & $61.59 \%$ \\
\hline \multirow{3}{*}{ Arbeitgeber } & öffentlich & 166 & $23.18 \%$ \\
& Nonprofit Org. & 45 & $6.28 \%$ \\
\hline
\end{tabular}

Die Daten dieser Befragung sind öffentlich zugänglich und weiter nutzbar (Willems et al. 2021b), ebenso wie alle folgenden grafischen Auswertungen sowie weitere Detailauswertungen (Willems et al. 2021a). In dieser Studie wird ein experimentelles Vorgehen in der Datenerhebung (Survey-Experiment) mit einer deskriptiven Analyse der Daten kombiniert. Um die erste Forschungsfrage zu beantworten (Erwartungshaltung von Bürgerinnen und Bürgern bzw. Kundinnen und Kunden gegenüber technologischen Neuerungen in der Leistungserbringung), analysieren wir die Mittelwerte der erhobenen Items.

Um die zweite Forschungsfrage zu beantworten (Einfluss des leistungserbringenden Sektors), wurde auf das experimentelle Vorgehen zur Datenerhebung aufgebaut (sample split). Die Treatment-Gruppe wurde zu Erwartungen in Bezug auf den öffentlichen Sektor befragt (Gruppe A), die Kontrollgruppe zu Erwartungen in Bezug auf den privatwirtschaftlichen Sektor (Gruppe B). Die Einteilung der Befragten in die Experimentalgruppen erfolgte randomisiert zu Beginn der Befragung. Dieses experimentelle Setting, bei dem die Befragten nicht in Bezug auf beide Sektoren antworten (sog. between-subject design), macht diese nicht darauf aufmerksam, dass Unterschiede zwischen öffentlichen und 
privatwirtschaftlichen Organisationen erwartet werden. Es ist daher geeignet, die zweite Forschungsfrage zu beantworten. Das gewählte Forschungsdesign testet konservativer auf Unterschiede, als wenn alle Befragten zu beiden Gruppen befragt worden wären. Dann hätten die Befragten möglicherweise stärker darauf geachtet, unterschiedliche Antworten für beide Gruppe zu geben und dabei eventuell generelle Unterschiede zwischen öffentlichem und privatem Sektor in ihre Einschätzung mit einbezogen, anstatt auf den Einsatz von Technologie zu fokussieren.

\section{Ergebnisse}

Ziel dieser Studie war es zunächst zu ergründen, welche Wirkungen Bürgerinnen und Bürger von neuen Technologien erwarten. Wichtig ist zu bedenken, dass es sich um eine Erwartungsabfrage handelt und nicht nach erlebten oder gewünschten Wirkungen gefragt wurde. Es ist aber davon auszugehen, dass erlebte Erfahrungen die Erwartungshaltung an Wirkungen von Technologien beeinflussen (Kaasinen et al. 2013).

Insgesamt zeigt sich, dass die Befragten so gut wie keine Erwartungen haben (vgl. Abb. 1). Leichte positive Veränderungen werden in Bezug auf die Effizienz der Leistungserbringung, die Erschwinglichkeit von Dienstleistungen (also deren Kosten für die Kundinnen und Kunden) und die Qualität der Leistungserbringung erwartet. In allen anderen abgefragten Aspekten (faire Behandlung, Sicherheit der Kundinnen und Kunden, allgemeine Zufriedenheit mit Dienstleistung, Transparenz gegenüber Kundinnen und Kunden, Datenschutz, ethisch verantwortungsvolle Behandlung, allgemeines Wohlbefinden der Kundinnen und Kunden) werden weder positive noch negative Veränderungen erwartet.

Abb. 1: Erwartung über Wirkung von neuen Technologien, gesamte Stichprobe ohne Trennung nach öffentlicher/privater Sektor

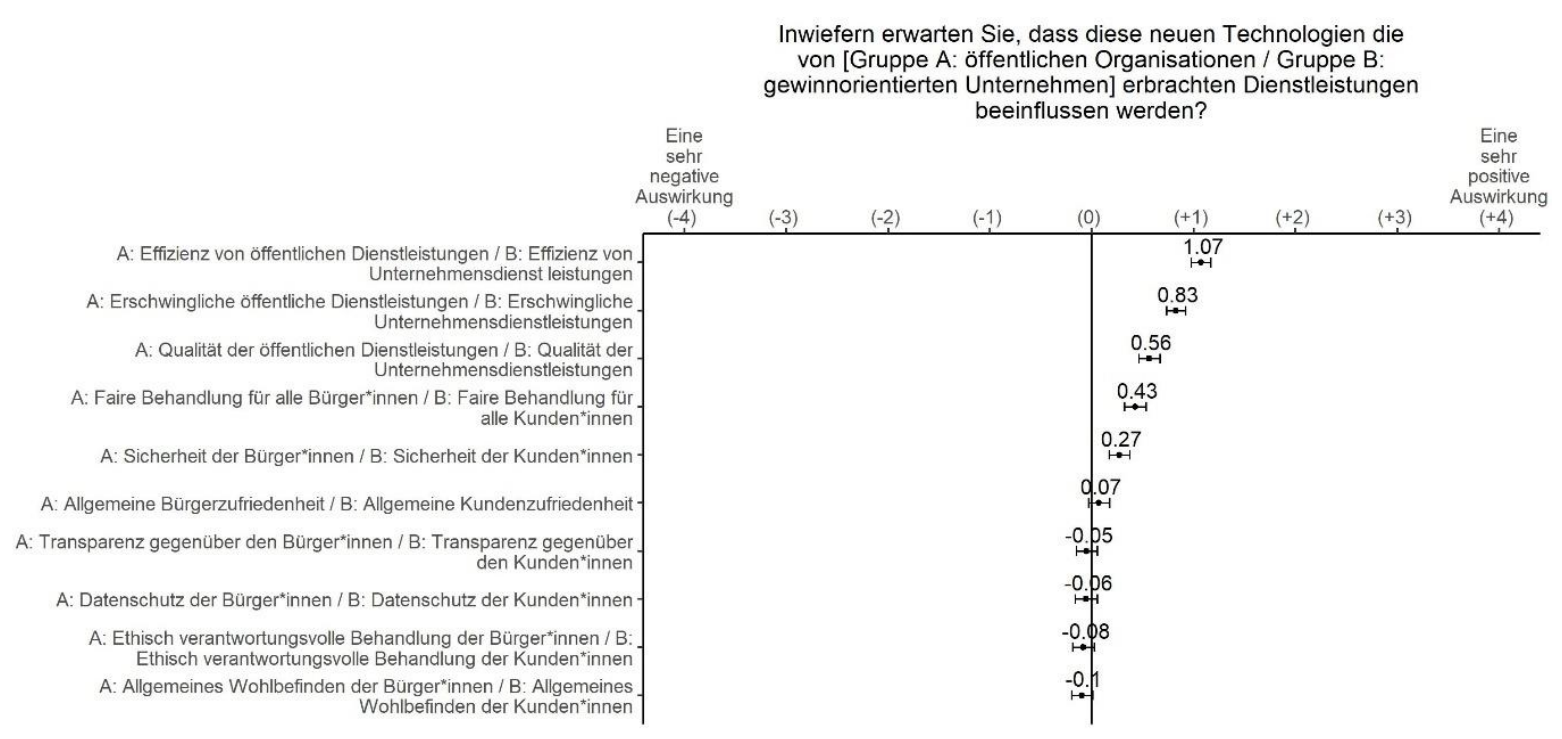

Legende: Mittelwerte und 95\%-Konfidenzintervalle, $\mathrm{N}=1.577$ 
Untersucht man diese Daten genauer darauf, ob die Befragten unterschiedliche Erwartungen in Bezug auf öffentliche oder private Dienstleistungen haben (vgl. Abb. 2), wird deutlich, dass die erwarteten Wirkungen bei gewinnorientierten Dienstleistungen etwas positiver eingeschätzt werden. Hier wird dem privatwirtschaftlichen Sektor insbesondere mehr Effizienz durch die Nutzung neuer Technologien zugeschrieben. In allen anderen Aspekten überschneiden sich die Erwartungen jedoch so stark, dass nicht von einer anderen Erwartungshaltung gesprochen werden kann.

Abb. 2: Erwartung über Wirkung von neuen Technologien, Stichprobe getrennt nach öffentlichen und privatwirtschaftlichen Dienstleistungen

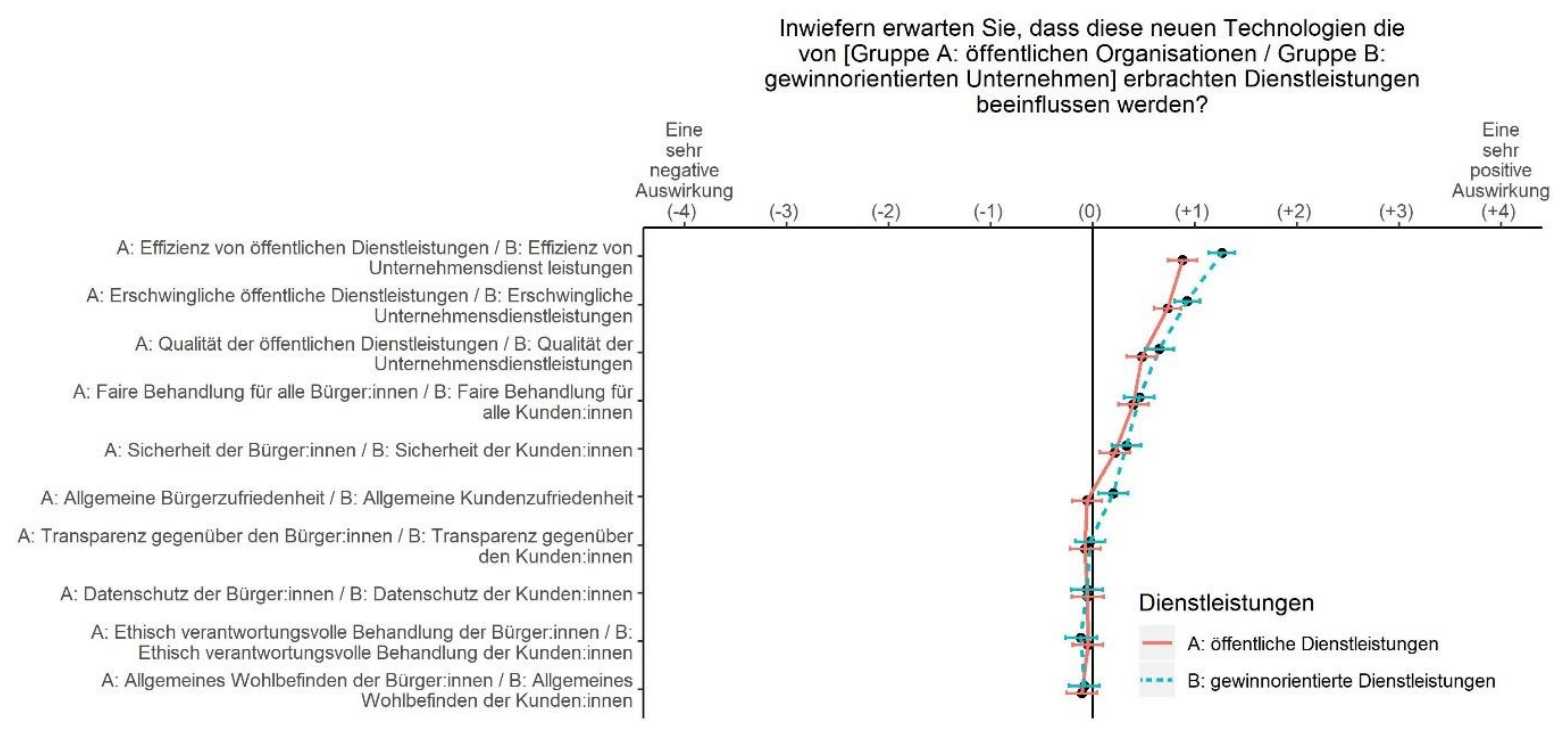

Legende: Mittelwerte und 95\%-Konfidenzintervalle, $\mathrm{N}=1.577$

Insgesamt herrscht also eine sehr geringe Erwartungshaltung in Bezug auf neue Technologien, die in öffentlichen Organisationen genutzt werden. Vielmehr scheinen Bürgerinnen und Bürger davon auszugehen, dass diese Technologien gar keinen Einfluss auf die Leistungserbringung der Verwaltung haben. Im Vergleich zu privatwirtschaftlichen Organisationen, in denen die erwartete Wirkung ebenfalls nahe null liegt, erwarten Bürgerinnen und Bürger von öffentlichen Organisationen sogar noch weniger. Man könnte argumentieren, dass dies daran liegt, dass Bürgerinnen und Bürger zu schlecht darüber informiert sind, was in öffentlichen Organisationen vor sich geht, welche Entwicklungen sich dort im Bereich Digitalisierung anbahnen und welche Wirkungen möglich wären.

Eine Aufsplittung der Stichprobe danach, ob die befragten Personen im privatwirtschaftlichen oder öffentlichen Sektor arbeiten, zeigt aber, dass selbst Mitarbeitende öffentlicher Organisationen die Wirkung neuer Technologien nicht anders einschätzen (vgl. Abb. 3). Deren Binnenperspektive scheint also nicht wesentlich von der Wahrnehmung externer Nutzerinnen und Nutzer (Mergel et al. 2018) 
abzuweichen. Ein Erklärungsansatz hierfür kann sein, dass die befragten Mitarbeiterinnen und Mitarbeiter im öffentlichen Sektor selbst keinerlei Kontakt zu ersten Anwendungsprojekten neuer Technologien haben, also eben gar keine internen Nutzerinnen und Nutzer sind. Dies würde aber bedeuten, dass die entsprechenden Technologieprojekte nach wie vor in kleinen Zirkeln besprochen werden und noch nicht in der Breite der Verwaltung angekommen sind. Ein anderer Erklärungsansatz wäre, dass selbst interne Nutzerinnen und Nutzer, also Mitarbeiterinnen und Mitarbeiter, die bereits Kontakt mit der Verwendung neuer Technologien gemacht haben, keine wesentlichen Veränderungen über die eigentliche Leistungserbringung heraus erwarten. Letztere Erklärung erscheint aber in Anbetracht der oben diskutierten Spannungen zwischen öffentlichen Werten, beispielsweise bei der Anwendung von algorithmusbasierten Assistenzsystemen, unwahrscheinlich.

Abb. 3: Erwartung über Wirkung von neuen Technologien auf öffentliche Leistungserbringung, Stichprobe getrennt nach Arbeitgeber/in

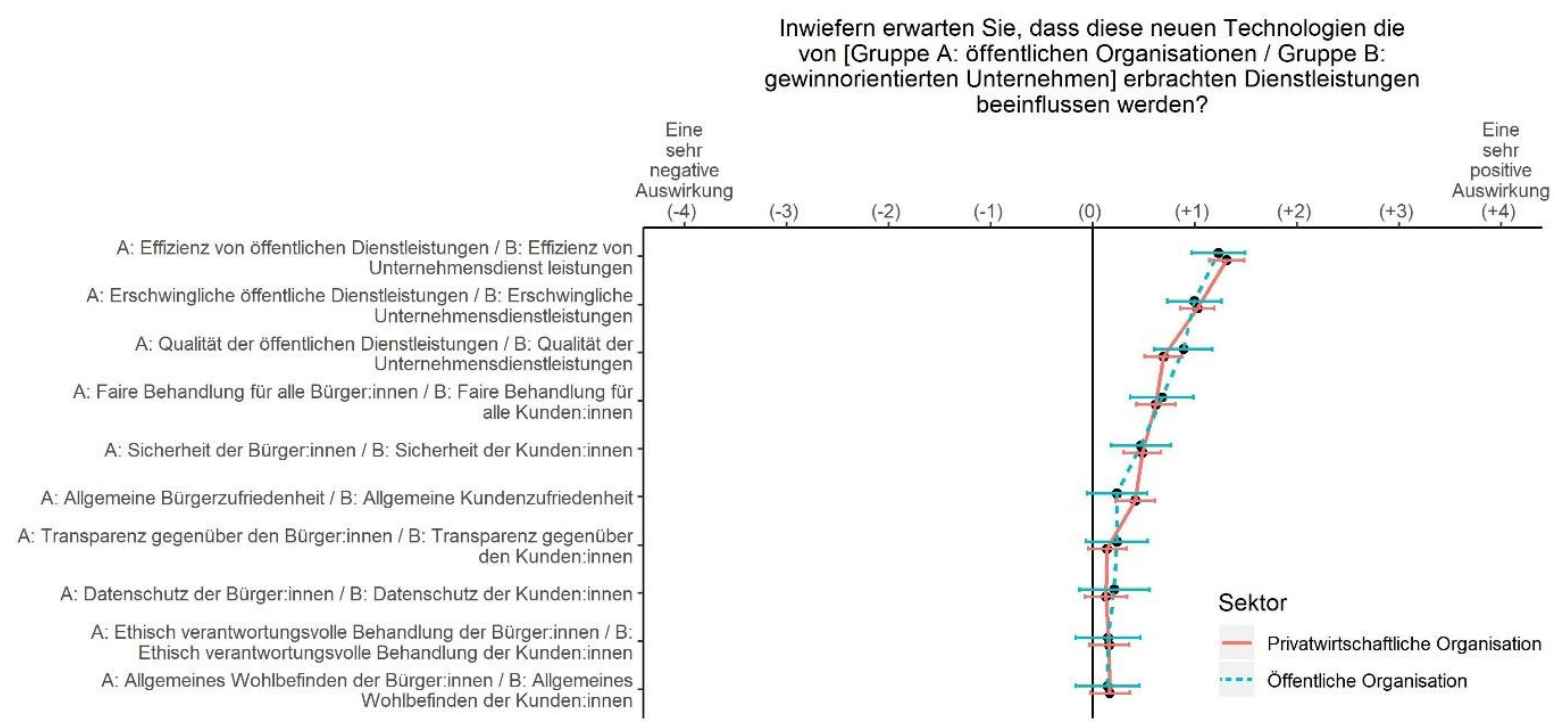

Legende: Mittelwerte und 95\%-Konfidenzintervalle, Teilstichprobe Angestellte in öffentlichen oder privatwirtschaftlichen Organisationen, $\mathrm{N}=607$

Neben dem Einfluss von Insiderwissen, also einer Beschäftigung in einer öffentlichen Organisation, scheinen auch demografische Variablen als Einflussfaktoren in Frage zu kommen. Eine Auswertung nach Altersgruppen der Befragten (vgl. Abb. 4) zeigt beispielsweise, dass Jüngere positivere Erwartungen an neue Technologien haben als Ältere. Insbesondere Menschen über 60 Jahren erwarten deutlich negativere Auswirkungen. Dies scheint auf deren Sozialisation mit Technologien, einer Gewöhnung an diese und entsprechende Kenntnisse und Fähigkeiten sowie damit verknüpfte Neugier oder Ängste zusammenzuhängen (Freese et al. 2006; Olphert und Damodaran 2013). Andere Studien, die den Anteil der täglichen Internetnutzung, den digitalen Kontakt mit Behörden oder die Nutzung von smarten Lösungen im Haushalt (Internet der Dinge) in Bezug auf das Alter der Nutzerinnen und Nutzer 
betrachten, bestätigen dieses Bild. Die tägliche Internetnutzung sowie die Nutzung des Internets der Dinge liegt bei über 65-Jährigen deutlich niedriger als in anderen Altersgruppen, ebenso sieht es mit der Nutzung von E-Government aus (Initiative D21 2020, S. 15; Statistisches Bundesamt 2021, 13, 20, 42). Auch stehen über 55-jährige Bürgerinnen und Bürger E-Government skeptischer gegenüber, sie haben Sicherheitsbedenken, glauben nicht an effizientere digitale Lösungen und haben Angst vor Fehlern (Initiative D21 2020, S. 38)Klicken oder tippen Sie hier, um Text einzugeben.Klicken oder tippen Sie hier, um Text einzugeben.. Ältere Offlinerinnen und Offliner können in der Digitalisierung schlichtweg keinen Nutzen für sich selbst erkennen (Kubicek 2019, S. 421). Diese Erkenntnisse dürften auch auf die Erwartungshaltung gegenüber den untersuchten neuen Technologien zutreffen.

Abb. 4: Erwartung über Wirkung von neuen Technologien, Stichprobe getrennt nach Altersgruppe

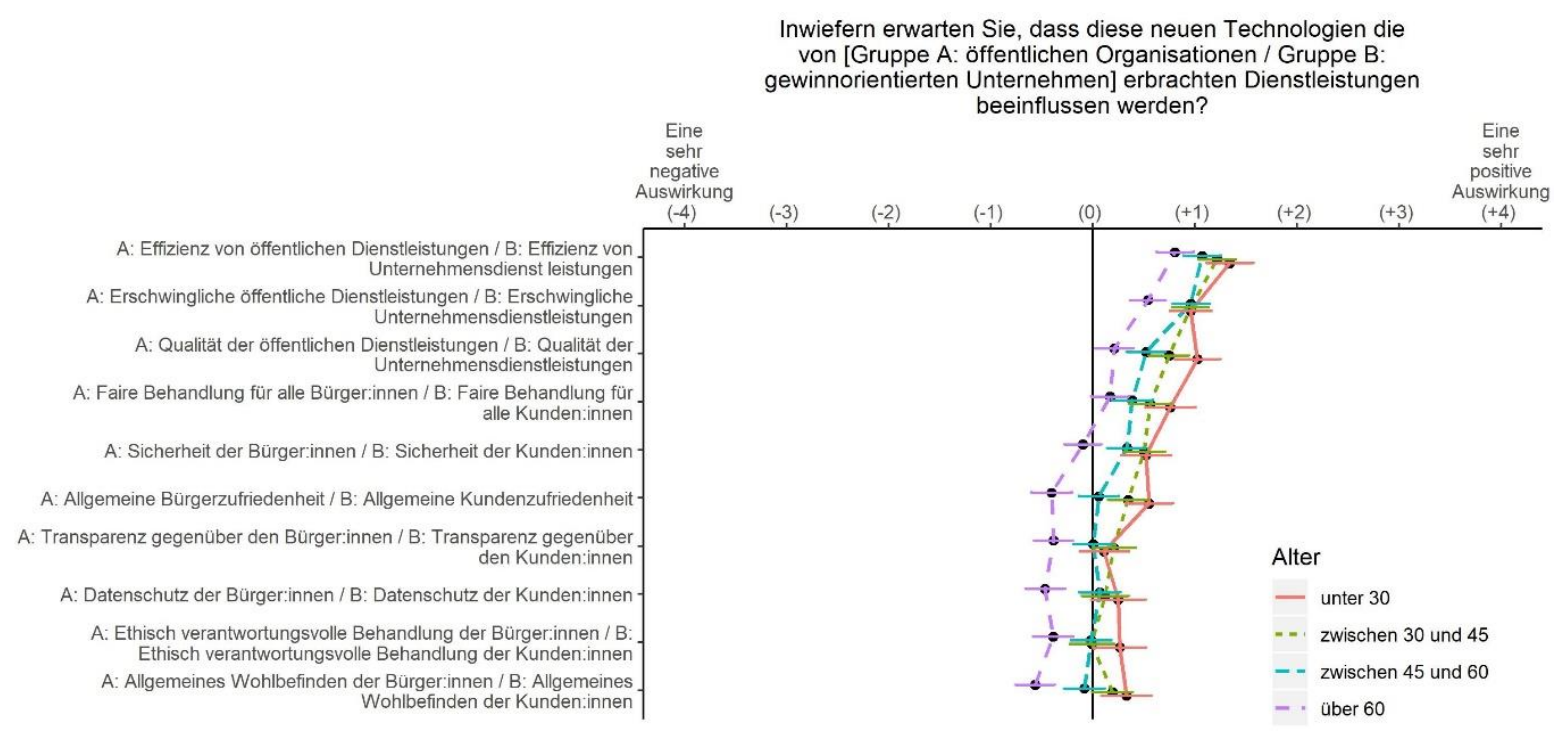

Legende: Mittelwerte und 95\%-Konfidenzintervalle, $\mathrm{N}=1.577$

Betrachtet man die mögliche Wirkung weiterer demografischer Einflussfaktoren, zeigen sich allerdings keine Effekte des Geschlechts, des Bildungsstandes oder eines Migrantinnen- und Migrantenstatus. Auch eine Auswertung der Daten in Bezug auf Unterschiede zwischen den Bundesländern zeigt keine systematischen Unterschiede. Ein oft vermuteter geringerer Digitalisierungsfortschritt in Ostdeutschland (Opiela 2019, 11, 25, 29, 38) und eine geringere Nutzung von E-Government durch Ostdeutsche (Statistisches Bundesamt 2021, S. 20) spiegelt sich in der Erwartungshaltung der Bürgerinnen und Bürger ebenfalls nicht wider (Abb. 5). 
Abb. 5: Erwartung über Wirkung von neuen Technologien, Stichprobe getrennt nach ost- und westdeutschen Bundesländern

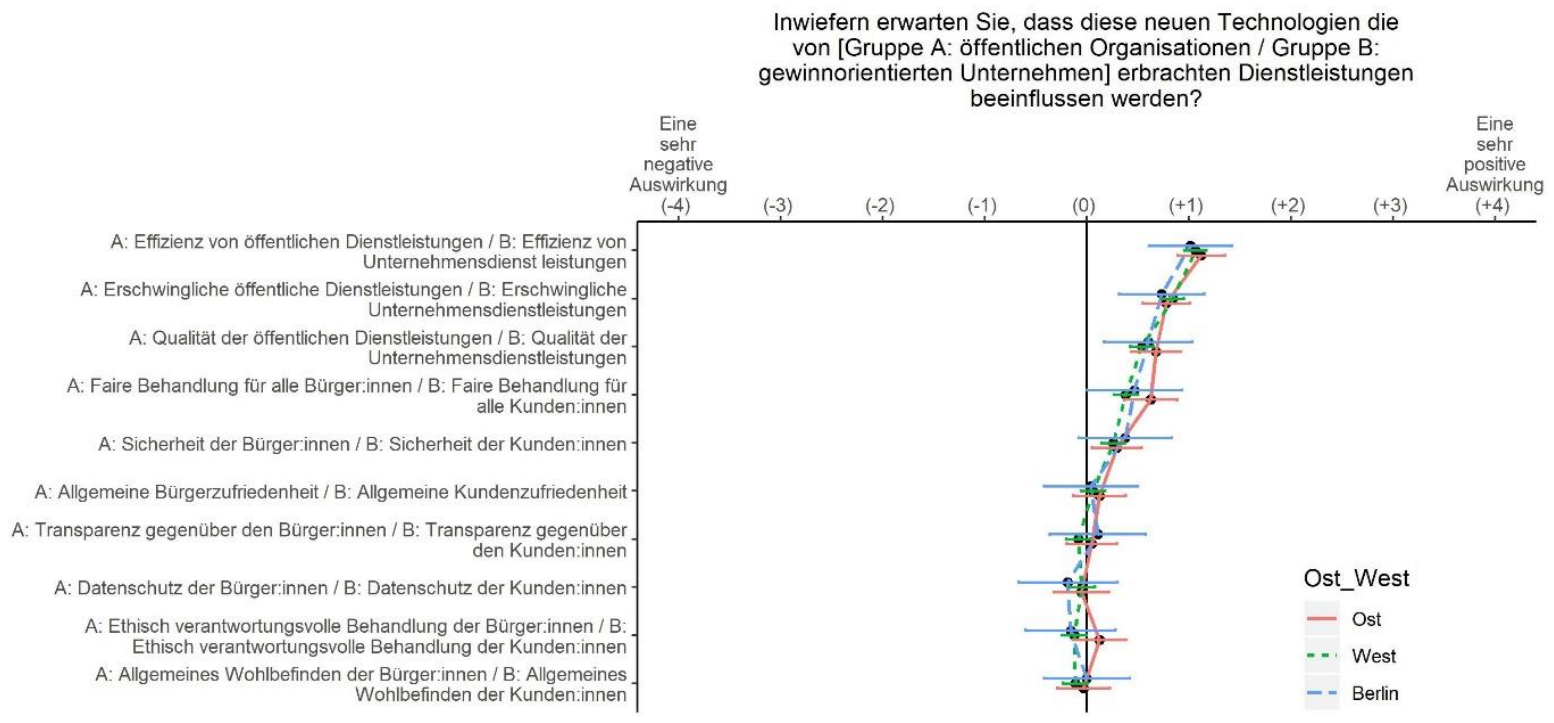

Legende: Mittelwerte und 95\%-Konfidenzintervalle, $\mathrm{N}=1.577$

Als Limitation sei angemerkt, dass auch methodische Schwächen zu den aufgeführten Ergebnissen beitragen können. Es erscheint möglich, dass das Antwortverhalten der Befragten um den Nullpunkt pendelt, weil sie erstens kein Verständnis davon haben, nach was sie gefragt wurden (neue Technologien). Es wurde in der Befragung zwar genannt, welche konkreten Technologien hierbei gemeint sind, diese aber nicht näher erklärt oder Beispiele genannt, um eine Beeinflussung der Befragten zu vermeiden. Diese vermeintliche Schwäche wird im folgenden Abschnitt ausführlicher diskutiert. Zweitens kann auch die Formulierung der Wirkungsdimensionen (Effizienz, erschwingliche Dienstleistungen, Qualität von Dienstleistungen usw.) zu breit und vage formuliert gewesen sein. Es scheint möglich, dass die Befragten schlicht nicht wussten, was Qualität oder Transparenz genau bedeutet und daher zu einer mittleren Antwortkategorie tendierten. Allerdings dürfte dies zumindest unter Mitarbeiterinnen und Mitarbeiter des öffentlichen Sektors weniger der Fall sein, weil diese mit dieser Terminologie vertraut sein müssten. Da ihr Antwortverhalten nicht wesentlich von dem der anderen Bürgerinnen und Bürger abweicht, dürften die Ergebnisse also dennoch valide sein. Zusätzlich validiert die Analyse des Einflusses von Alter unsere Ergebnisse, denn sie entspricht bisherigen Befunden in der Literatur.

\section{Fazit und Schlussfolgerungen}

Die Daten dieser Studie legen insgesamt nahe, dass Bürgerinnen und Bürger bzw. Kundinnen und Kunden keine deutliche Wirkung von der Anwendung neuer Technologien, wie etwa Algorithmen, künstliche Intelligenz oder Blockchains, erwarten. Das gilt für alle erfragten Wirkungsdimensionen, die 
sich an gesellschaftlichen Werten ausrichten. Lediglich in Bezug auf die Effizienz der Dienstleistungserbringung versprechen sich die Befragten leichte positive Effekte. Die Frage ist also, ob es bei der Einführung dieser Technologien über den Selbstzweck hinaus nicht nur um reine Effizienz geht, oder ob diese, wie medial diskutiert wird, auch zu gesellschaftlichen Verbesserungen in Bezug auf Transparenz, Fairness oder ethische Standards dienen. Dies sollte insbesondere für die öffentliche Leistungserbringung gelten. Wie unsere Daten aber zeigen, sehen wir keinen deutlichen Unterschied zwischen gewinnorientierten Dienstleistungen und Technologieprojekten in der Verwaltung.

Wenn aber weitergehende Wirkungen erzielt werden (sollen) und Expertinnen und Experten solche Wirkungen auch planen und erwarten, dann ist die Frage, warum die Ergebnisse für die Bürgerinnen und Bürger hier abweichen. Dafür gibt es unserer Ansicht nach zwei Erklärungsansätze: (1) Sie wissen es nicht besser oder (2) sie haben genau diese Erfahrung der Wirkungslosigkeit gemacht.

Für die erste Erklärung - die Bürgerinnen und Bürger können Wirkungen nicht abschätzen - sprechen verschiedene Argumente. Wie Olsson (2014) darlegt, basieren Erwartungshaltungen von potenziellen Nutzerinnen und Nutzern einer Technologie auf eigenen Wünschen und sozialen oder gesellschaftlichen Normen. Eigene Wünsche können sich aber nur entwickeln, wenn auch Erfahrungen mit dieser oder ähnlichen Technologien vorliegen. Diese Erfahrungen können eigene sein, Erfahrungen anderer oder auf Hören-Sagen basieren (Olsson 2014, S. 1959). Wenn die besprochenen Technologien bisher aber noch gar nicht in der Lebensrealität der Bürgerinnen und Bürger angekommen sind, sie sie also nicht kennen, können sie auch keine Wirkungen abschätzen. Da die analysierten Technologien bisher nach wie vor eher in Pilotprojekten angewendet werden, z.B. werden digitale Assistenzsysteme in Verwaltung bisher wenig genutzt (Initiative D21 2020, S. 44), dürfte das für die Mehrheit der Bürgerinnen und Bürger gelten. Es sei aber hinzugefügt, dass sich Wirkungen auf die hier untersuchten Werte erst langfristig einstellen dürften und nicht kurzfristig beobachtbar sind (Fukumoto und Bozeman 2019, S. 643).

Fehlende Erfahrung bedeutet dennoch nicht, dass es nicht trotzdem eine entsprechende Erwartungshaltung geben kann, denn wie Kurniawan et al. (2018) zeigen, basieren Erwartungen von Bürgerinnen und Bürgern in Bezug auf Innovationen im öffentlichen Sektor auch auf Bedürfnissen, die momentan nicht erfüllt werden. Hjortskov (2020) und Favero und Kim (2020) unterscheiden Erfahrungen aufgrund dieser unterschiedlichen Herkunft in vorhersagende und normative Erwartungen. Auf welchen dieser Erwartungen die Aussagen der Teilnehmenden an dieser Studie beruhen, kann nicht näher bestimmt, jedoch ist davon auszugehen, dass vorhersagende Erfahrungen auf Basis mangelnder Anwendungen eher gering ausfallen. 
Es schließt sich die Frage an, ob es sinnvoll ist, Bürgerinnen und Bürger zu befragen, wenn diese über den Untersuchungsgegenstand gar nicht Bescheid wissen (können). Wir erkennen so, dass ein mehr an Kommunikation nötig ist, um den Bürgerinnen und Bürger schon allein aus Legitimationsgründen stärker zu vermitteln, welche größeren Effekte sich die Verwaltung von KI, Blockchain und Co. verspricht. Das Erwartungsmanagement muss hier allerdings auch realistisch bleiben und darf keine Erwartungen wecken, die nicht erfüllt werden können. Im Sinne von Open Government und Nutzerorientierung kann und sollte hier auch über bloße Kommunikation hinaus gegangen werden und Bürgerinnen und Bürger bzw. Kundinnen und Kunden direkt in die Planung und Implementation solcher Technologien eingebunden werden (z.B.: participatory prototyping).

Für die zweite Erklärung - die Bürgerinnen und Bürger wissen, dass es (bisher) keine Wirkungen gibt - spricht zum einen, dass selbst Verwaltungsmitarbeitende keine Wirkungen von den neuen Technologien für die öffentliche Leistungserbringung erwarten. Sie unterscheiden sich in ihrer Einschätzung als „Insider“ nicht von den Bürgerinnen und Bürger. Wie besprochen, scheint es möglich, dass der Verbreitungsgrad der besprochenen Technologien so niedrig ist, dass selbst durchschnittliche Verwaltungsmitarbeitende keinen Kontakt zu diesen haben oder aber über entsprechende Projekte innerhalb der Verwaltungen nicht kommuniziert wird. Möglicherweise ist deren Fähigkeit, Wirkungen von Technologie einzuschätzen, daher auch zu gering (impact awareness als eine digitale Kompetenz: Hofmann und Ogonek 2018).

Wenn es ein Kommunikationsdefizit gibt, tut die Verwaltung gut daran, hier mehr interne Teilhabe zu ermöglichen, Informationen stärker zu streuen, mehr formelles und informelles Lernen in dieser Hinsicht zu ermöglichen. Wenn Verwaltungsmitarbeitende nicht erklären können, warum es Investitionen in neue Technologien geben muss, kann man dies von den Bürgerinnen und Bürgern auch nicht erwarten. Wenn es aber nicht an mangelnder Kommunikation liegt, sondern selbst Mitarbeiterinnen und Mitarbeiter der öffentlichen Verwaltungen nicht überzeugt sind, dass neue Technologien keine gesellschaftliche Wirkung entfalten werden, dann muss ernsthaft in Frage gestellt werden, ob hier weiter investiert werden sollte. Die Verwaltung muss nicht jedem technologischen Hype hinterherjagen, wenn entsprechende Technologien keinen gesellschaftlichen Mehrwert bieten. Die Euphorie, mit der einige Politikerinnen und Politiker, Beraterinnen und Berater aber auch Wissenschaftlerinnen und Wissenschaftler hinsichtlich der gesellschaftlichen Chancen eines Einsatzes dieser Technologien in der Verwaltung begegnen, scheint insofern nicht gerechtfertigt.

Neben diesen scheinbar fehlenden Erwartungshaltungen von Bürgerinnen und Bürgern in Bezug auf den Effekt neuer Technologien zeigt unsere Studie auch eine fehlende Differenzierung zwischen öffentlichem und privatem Sektor. Zugespitzt formuliert: Bürgerinnen und Bürger erwarten von Technologie keine andere Wirkung, wenn der Staat diese verwendet als wenn es ein 
privatwirtschaftliches Unternehmen tut. Man könnte argumentieren, dass dies nach 30 Jahren Reformen des sogenannten New Public Management und einer stärkeren Fokussierung der Verwaltung auf Effizienz und der Orientierung an den Bedürfnissen der Kundinnen und Kunden nicht verwundert und sogar wünschenswert ist. Wir haben in dieser Studie allerdings nicht Erwartungshaltungen an Interaktion mit Kundinnen und Kunden im Allgemeinen analysiert, sondern spezifisch nach der Wirkung neuer Technologien gefragt. Zumindest in dieser Hinsicht scheinen Bürgerinnen und Bürger in ihrer Rolle als Kundinnen und Kunden gegenüber Verwaltung und Unternehmen die gleichen Maßstäbe anzulegen.

Das erscheint uns aber insofern als problematisch, als die Verwaltung mit ihren Entscheidungen viel stärker in das Leben der Bürger eingreifen darf als privatwirtschaftliche Unternehmen. Zudem können Bürgerinnen und Bürger Verwaltungsleistungen im Unterschied zu privatwirtschaftlichen nicht einfach beim Konkurrenten einkaufen, wenn ihnen etwas nicht gefällt oder ihre Rechte und Werte verletzt werden. Die Public-Value-Theorie geht davon aus, dass die hier untersuchten Werte daher politische und Verwaltungsentscheidungen treiben sollten (Fukumoto und Bozeman 2019, S. 638). Diese Werte stellen einen normativen Konsens dar, welcher Rechte und Unterstützung sich Bürgerinnen und Bürger sicher sein können und auf welchen Prinzipien Verwaltungshandeln basiert (Bozeman 2007, S. 13). Im Umkehrschluss müssten also auch entsprechende Ergebnisse ersichtlich werden.

\section{Literatur}

Alruwaie, Mubarak; El-Haddadeh, Ramzi; Weerakkody, Vishanth (2020): Citizens' continuous use of eGovernment services: The role of self-efficacy, outcome expectations and satisfaction. In: Government Information Quarterly 37 (3), S. 101485. DOI: 10.1016/j.giq.2020.101485.

Andersen, Kim Normann; Medaglia, Rony; Vatrapu, Ravi; Henriksen, Helle Zinner; Gauld, Robin (2011): The forgotten promise of e-government maturity: Assessing responsiveness in the digital public sector. In: Government Information Quarterly 28 (4), S. 439-445. DOI: 10.1016/j.giq.2010.12.006.

Anthes, Gary (2015): Estonia. In: Commun. ACM 58 (6), S. 18-20. DOI: 10.1145/2754951.

Behnke, Nathalie (2005): Alte und neue Werte im öffentlichen Dienst. In: Bernhard Blanke, Stephan von Bandemer, Frank Nullmeier und Göttrik Wewer (Hg.): Handbuch zur Verwaltungsreform. Wiesbaden: VS Verlag für Sozialwissenschaften, S. 243-251.

Boudreau, Christian; Bernier, Luc (2017): The implementation of integrated electronic service delivery in Quebec: the conditions of collaboration and lessons. In: International Review of Administrative Sciences 83 (3), S. 602-620. DOI: 10.1177/0020852315598215.

Bozeman, Barry (2007): Public values and public interest. Counterbalancing economic individualism. Washington, D.C: Georgetown University Press (Public Management and Change Series). Online verfügbar unter http://site.ebrary.com/lib/academiccompletetitles/home.action.

Cavalheiro, Gabriel Marcuzzo do Canto; Joia, Luiz Antonio (2016): E-Government Technology Transfer: A Case Study of the Implementation of a European Patent Management System in Brazil. In: Public Admin. Dev. 36 (3), S. 215-231. DOI: 10.1002/pad.1753. 
Dekker, Rianne; van den Brink, Puck; Meijer, Albert (2020): Social media adoption in the police: Barriers and strategies. In: Government Information Quarterly 37 (2), S. 101441. DOI: 10.1016/j.giq.2019.101441.

Engelmann, Jan; Puntschuh, Michael (2020): KI IM BEHÖRDENEINSATZ: ERFAHRUNGEN UND EMPFEHLUNGEN. Hg. v. Kompetenzzentrum für Öffentliche IT. Online verfügbar unter https://www.oeffentliche-

it.de/documents/10181/14412/Best+Practices+beim+Einsatz+K\%C3\%BCnstlicher+Intelligenz+in+der $+\% \mathrm{C} 3 \%$ B6ffentlichen+Verwaltung.

Fanta, Alexander (2020): Datenschutzbehörde stoppt Jobcenter-Algorithmus. In: Netzpolitik.org, 21.08.2020. Online verfügbar unter https://netzpolitik.org/2020/oesterreich-ams-datenschutzbehoerdestoppt-jobcenter-algorithmus/.

Favero, Nathan; Kim, Minjung (2020): Everything Is Relative: How Citizens Form and Use Expectations in Evaluating Services. In: Journal of Public Administration Research and Theory. DOI: 10.1093/jopart/muaa048.

Fischer, Caroline; Kraus, Sascha (2020): Digitale Transparenz. In: Tanja Klenk, Frank Nullmeier und Göttrik Wewer (Hg.): Handbuch Digitalisierung in Staat und Verwaltung. Wiesbaden: Springer Fachmedien Wiesbaden, S. 159-170.

Franke, Nikolaus; Keinz, Peter; Klausberger, Katharina (2013): “Does This Sound Like a Fair Deal?”: Antecedents and Consequences of Fairness Expectations in the Individual's Decision to Participate in Firm Innovation. In: Organization Science 24 (5), S. 1495-1516. DOI: 10.1287/orsc.1120.0794.

Freese, Jeremy; Rivas, Salvador; Hargittai, Eszter (2006): Cognitive ability and Internet use among older adults. In: Poetics 34 (4-5), S. 236-249. DOI: 10.1016/j.poetic.2006.05.008.

Fukumoto, Eriko; Bozeman, Barry (2019): Public Values Theory: What Is Missing? In: The American Review of Public Administration 49 (6), S. 635-648. DOI: 10.1177/0275074018814244.

Große Starmann, Carsten; Wiedemann, Mario (2017): Digitale Transformation der Verwaltung. Empfehlungen für eine gesamtstaatliche Strategie. Hg. v. Bertelsmann Stiftung. Online verfügbar unter https://www.bertelsmann-

stiftung.de/fileadmin/files/Projekte/Smart_Country/DigiTransVerw_2017_final.pdf.

Guggenmos, Florian; Lockl, Jannik; Rieger, Alexander; Fridgen, Gilbert (2019): Blockchain in der öffentlichen Verwaltung. In: Informatik Spektrum 42 (3), S. 174-181. DOI: 10.1007/s00287-01901177-y.

Heuermann, Roland; Engel, Andreas; Lucke, Jörn von (2018): Digitalisierung: Begriff, Ziele und Steuerung. In: Roland Heuermann, Matthias Tomenendal und Christian Bressem (Hg.): Digitalisierung in Bund, Ländern und Gemeinden, Bd. 2014. Berlin, Heidelberg: Springer Berlin Heidelberg, S. 9-50.

Hjortskov, Morten (2020): Interpreting expectations: Normative and predictive expectations from the citizens' viewpoint. In: JBPA 3 (1). DOI: 10.30636/jbpa.31.72.

Hofmann, Sara; Ogonek, Nadine (2018): Different But Still The Same? How Public And Private Sector Organisations Deal with New Digital Competences. In: The Electronic Journal of eGovernment 16 (2), S. 127-135.

Initiative D21 (2020): eGovernment MONITOR 2020. Staatliche Digitalangebote - Nutzung und Akzeptanz in Deutschland, Österreich und der Schweiz. 1. Auflage. Berlin: Initiative D21. 
Jørgensen, Torben Beck; Bozeman, Barry (2007): Public Values. In: Administration \& Society 39 (3), S. 354-381. DOI: 10.1177/0095399707300703.

Kaasinen, Eija; Kymäläinen, Tiina; Niemelä, Marketta; Olsson, Thomas; Kanerva, Minni; Ikonen, Veikko (2013): A User-Centric View of Intelligent Environments: User Expectations, User Experience and User Role in Building Intelligent Environments. In: Computers 2 (1), S. 1-33. DOI:

10.3390/computers2010001.

Kennedy, Helen; Elgesem, Dag; Miguel, Cristina (2017): On fairness. In: Convergence 23 (3), S. 270288. DOI: $10.1177 / 1354856515592507$.

Köver, Chris (2019): Streit um den AMS-Algorithmus geht in die nächste Runde. In: Netzpolitik.org, 10.10.2019. Online verfügbar unter https://netzpolitik.org/2019/streit-um-den-ams-algorithmus-gehtin-die-naechste-runde/.

Köver, Chris (2020): Gericht macht Weg für den AMS-Algorithmus wieder frei, 22.12.2020. Online verfügbar unter https://netzpolitik.org/2020/automatisierte-entscheidungen-gericht-macht-weg-fuerden-ams-algorithmus-wieder-frei/.

Kubicek, Herbert (2019): Digitalisierung und demografischer Wandel. In: Hans-Henning Lühr, Roland Jabkowski und Sabine Smentek (Hg.): Handbuch Digitale Verwaltung. 1. Aufl.: KSV Verwaltungspraxis, S. 413-430.

Kurniawan, Jude Herijadi; Ong, Corinne; Cheah, Lynette (2018): Examining values and influences affecting public expectations of future urban mobility: A Singapore case study. In: Transport Policy 66, S. 66-75. DOI: 10.1016/j.tranpol.2017.12.014.

Linders, Dennis; Liao, Calvin Zhou-Peng; Wang, Cheng-Ming (2018): Proactive e-Governance: Flipping the service delivery model from pull to push in Taiwan. In: Government Information Quarterly 35 (4), 68-76. DOI: 10.1016/j.giq.2015.08.004.

Lindgren, Ida; Madsen, Christian Østergaard; Hofmann, Sara; Melin, Ulf (2019): Close encounters of the digital kind: A research agenda for the digitalization of public services. In: Government Information Quarterly 36 (3), S. 427-436. DOI: 10.1016/j.giq.2019.03.002.

Mergel, Ines (2019): Digitale Transformation als Reformvorhaben der deutschen öffentlichen Verwaltung. In: der moderne staat 12 (1-2019), S. 162-171. DOI: 10.3224/dms.v12i1.09.

Mergel, Ines; Kattel, Rainer; Lember, Veiko; McBride, Keegan (2018): Citizen-oriented digital transformation in the public sector. In: Marijn Janssen, Soon Ae Chun, Vishanth Weerakkody, Anneke Zuiderwijk und Charles C. Hinnant (Hg.): Proceedings of the 19th Annual International Conference on Digital Government Research: Governance in the Data Age. dg.o '18: 19th Annual International Conference on Digital Government Research. Delft The Netherlands, 300520180106 2018. New York, NY, USA: ACM, S. 1-3.

Olphert, Wendy; Damodaran, Leela (2013): Older people and digital disengagement: a fourth digital divide? In: Gerontology 59 (6), S. 564-570. DOI: 10.1159/000353630.

Olsson, Thomas (2014): Layers of user expectations of future technologies. In: Matt Jones, Philippe Palanque, Albrecht Schmidt und Tovi Grossman (Hg.): CHI '14 Extended Abstracts on Human Factors in Computing Systems. CHI '14: CHI Conference on Human Factors in Computing Systems. Toronto Ontario Canada, 260420140105 2014. New York, NY, USA: ACM, S. 1957-1962.

Opiela, Nicole (2019): Deutschland-Index der Digitalisierung 2019. Hg. v. Kompetenzzentrum Öffentliche IT.

Puntschuh, Michael; Fetic, Lajla (2020): Handreichung für die digitale Verwaltung. 
Róiste, Mairéad de (2013): Bringing in the users: The role for usability evaluation in eGovernment. In: Government Information Quarterly 30 (4), S. 441-449. DOI: 10.1016/j.giq.2013.05.007.

Schmid, Andreas (2019): Verwaltung, eGovernment und Digitalisierung. Wiesbaden: Springer Fachmedien Wiesbaden.

Schulze, Anna; Bender, Juliane; Webe, Fabian (2019): Blockchain in der Bundesverwaltung: Machbarkeitsanalyse. In: VM 25 (2), S. 83-89. DOI: 10.5771/0947-9856-2019-2-83.

Scupola, Ada; Zanfei, Antonello (2016): Governance and innovation in public sector services: The case of the digital library. In: Government Information Quarterly 33 (2), S. 237-249. DOI: 10.1016/j.giq.2016.04.005.

Statistisches Bundesamt (2021): Wirtschaftsrechnungen. Private Haushalte in der Informationsgesellschaft - Nutzung von Informations- und Kommunikationstechnologien. Online verfügbar unter https://www.destatis.de/DE/Themen/Gesellschaft-Umwelt/Einkommen-KonsumLebensbedingungen/IT-Nutzung/Publikationen/Downloads-IT-Nutzung/private-haushalte-ikt2150400207004.pdf?_blob=publicationFile.

Twizeyimana, Jean Damascene; Andersson, Annika (2019): The public value of E-Government - A literature review. In: Government Information Quarterly 36 (2), S. 167-178. DOI: 10.1016/j.giq.2019.01.001.

Vanderelst, Dieter; Willems, Jurgen (2020): Can We Agree on What Robots Should be Allowed to Do? An Exercise in Rule Selection for Ethical Care Robots. In: Int J of Soc Robotics 12 (5), S. $1093-$ 1102. DOI: 10.1007/s12369-019-00612-0.

Wagner, Tobias (2018): Digitalisierung im Bürgerkontakt - Ein Streifzug durch die Möglichkeiten und Hürden des Datenaustauschs zwischen Behörden und Bürgern. In: Christian Bär, Thomas Grädler und Robert Mayr (Hg.): Digitalisierung im Spannungsfeld von Politik, Wirtschaft, Wissenschaft und Recht. Berlin, Heidelberg: Springer Berlin Heidelberg, S. 543-552.

Willems, Jurgen; Fischer, Caroline; van den Bergh, Joachim (2021a): Citizens' Expected Impact of New Technologies in Public and For-profit Services. Auswertungen und Abbildungen. Online verfügbar unter https://doi.org/10.6084/m9.figshare.13693693.

Willems, Jurgen; Fischer, Caroline; van den Bergh, Joachim (2021b): Citizens' Expected Impact of New Technologies in Public and For-profit Services. Datensatz. Online verfügbar unter https://osf.io/vqtgc/.

Willems, Jurgen; van den Bergh, Joachim; Viaene, Stijn (2017): Smart City Projects and Citizen Participation: The Case of London. In: René Andeßner, Dorothea Greiling und Rick Vogel (Hg.): Public Sector Management in a Globalized World. Wiesbaden: Springer Fachmedien Wiesbaden, S. 249-266.

Woldaregay, Ashenafi Zebene; Henriksen, André; Issom, David-Zacharie; Pfuhl, Gerit; Sato, Keiichi; Richard, Aude et al. (2020): User Expectations and Willingness to Share Self-Collected Health Data. In: Studies in health technology and informatics 270, S. 894-898. DOI: 10.3233/SHTI200290. 